\title{
Unilateral Paramedian Transpedicular Approach for Repair of Anterior Transdural Spinal Cord Herniation: Report of a Case and Literature Review
}

\author{
Manish K. Kasliwal, John E. O’toole, Harel Deutsch \\ Department of Neurosurgery, Rush University Medical Center, Chicago, IL, USA
}

\begin{abstract}
Idiopathic spinal cord herniation is a rare but potentially treatable cause of thoracic myelopathy. The rarity and variable clinical presentation often results in missed diagnosis and delay in treatment. Posterior midline approach with laminectomy has been the most common approach performed for spinal cord herniation in cases described in the literature. A posterior approach is limited by the danger of retracting the spinal cord and difficulty visualizing the dural defect. Considering the anterior location of the dural defect, a posterolateral allows for a more ventral view without spinal cord manipulation. We report a rare case of idiopathic spinal cord herniation which was managed by unilateral paramedian transpedicular approach with an excellent clinical outcome.
\end{abstract}

Key Words: Spinal cord herniation, Surgery, Management, Thoracic spine

\section{Introduction}

Idiopathic spinal cord herniation is a rare but potentially treatable cause of thoracic myelopathy. The condition's rarity and variable clinical presentation can often result in missed diagnosis and delay in treatment [1,2]. Though pathogenesis and natural history remains uncertain, there is no differences in opinion over the need of surgery especially for symptomatic patients [3-9]. Posterior midline approach with laminectomy has been the most common approach performed for spinal cord herniation in cases described in the literature $[1,2,8]$. Considering the anterior location of the dural defect, posterolateral approaches allow for better ventral visualization without retraction on the spinal cord $[4,6]$. We report a rare case of idiopathic spinal cord herniation which was managed by unilateral paramedian transpedicular approach with an excellent clinical outcome.

\section{Case Report}

A 48-year-old female presented with two year history of back pain and progressive difficultly walking. She gradually developed increasing numbness in both lower limbs over one year. She also developed increasing urinary retention. There was no history of trauma/fever or any other systemic disease. She was evaluated by an orthopedic spine surgeon and was referred to a physician following a negative lumbar magnetic resonance imaging (MRI). Neurological examination revealed presence of weakness in bilateral lower extremities (4-/5, Medical Research Council $[\mathrm{MRC}]$ grade) and asymmetrical sensory loss from $\mathrm{T} 8$ below consistent with a diagnosis of thoracic myelopathy (Nurick grade 4). Persistent symptoms with worsening gait imbalance led us to perform of thoracic spine MRI which surprisingly revealed presence of a focal anterior kink of the thoracic cord at the T7-T8 level (Fig.

Received Feb 27, 2011; Revised Mar 24, 2011; Accepted Apr 8, 2011

Corresponding author: Manish K. Kasliwal, MD, MCh

Department of Neurosurgery, Rush University Medical Center,

1725 W Harrison Street, Suite 1115, Chicago, IL 60612, USA

Tel: +1-312-942-6644, Fax: +1-312-942-2176, E-mail: manish_kasliwal@rush.edu

Copyright (C) 2012 by Korean Society of Spine Surgery

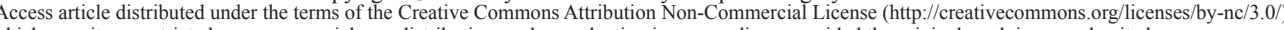
which permits unrestricted non-commercial use, distribution, and reproduction in any medium, provided the original work is properly cited. Asian Spine Journal • pISSN 1976-1902 eISSN 1976-7846 
1). The spinal cord at $T 7 / 8$ level was narrow and deformed without any definite $\mathrm{T} 2$ signal abnormality with prominence of the dorsal subarachnoid space. The MRI imaging was suspicious of a diagnosis of anterior transdural spinal cord herniation (Fig. 1). Surgical management was planned and a unilateral right paramedian incision was used to perform a transpedicular exposure due to slight eccentric location of the spinal cord herniation to the right (Fig. 1). Intraoperative neuromonitoring was performed recording somatosensory evoked potentials (SSEPs) and motor evoked potentials
(MEPs). T7/T8 pediculotomy was performed with partial costotransversectomy to provide the optimal anterolateral trajectory. A paramedian right dura incision was made, providing exquisite visualization of the anterior dural defect with the herniated spinal cord (Fig. 2). Using a Woodson dissector, the spinal cord was reduced back into the thoracic dura. The SSEP's were normal throughout the procedure. There was transient reduction in MEP amplitude during mobilization of the spinal cord to reduce the herniation. The MEP amplitude returned to baseline status an hour

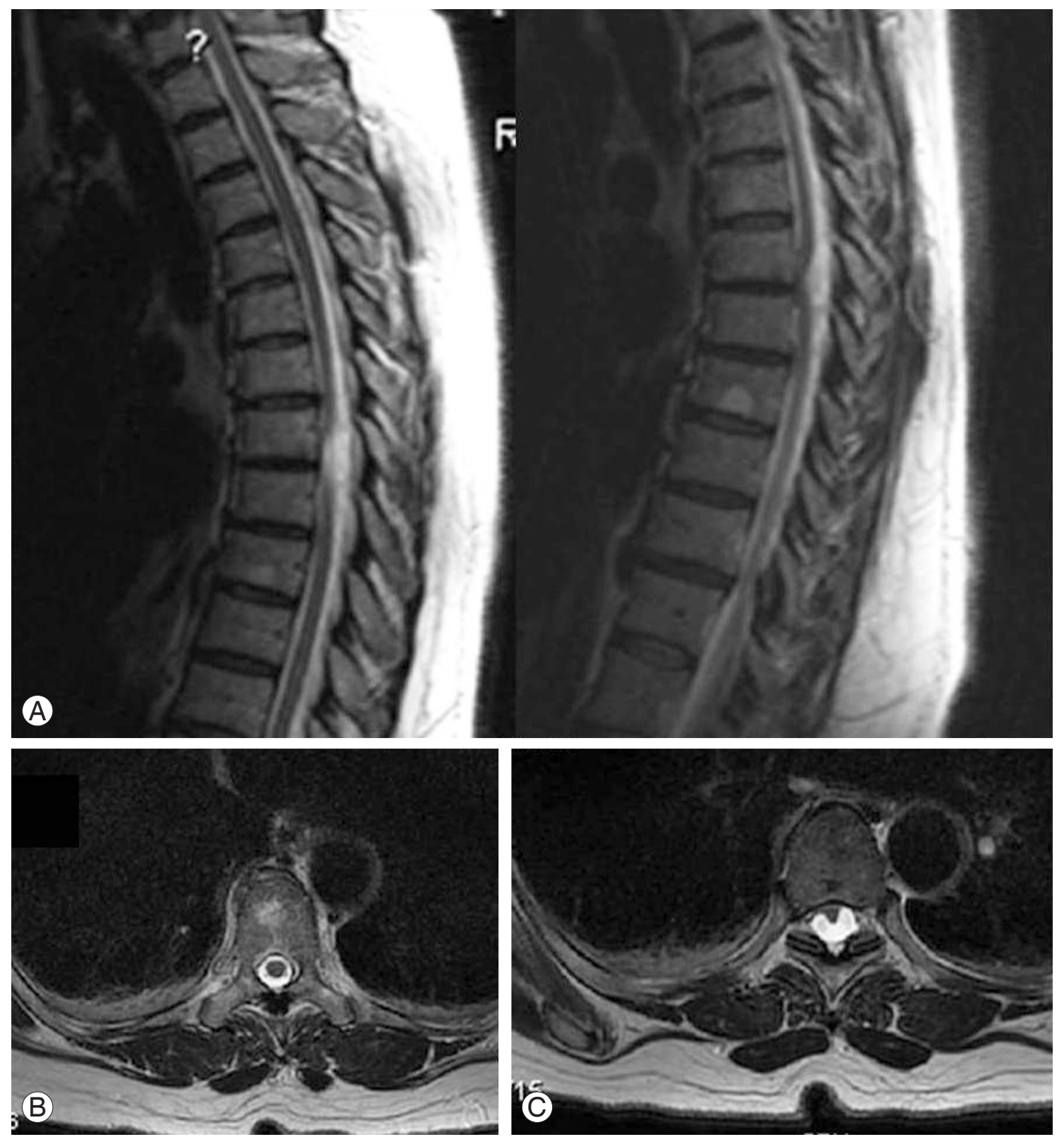

Fig. 1. T2W sagittal magnetic resonance imaging (MRI) (A) showing presence of a focal anterior C-shaped kink of the thoracic cord at the T7-T8 level with almost disappearance of the cord at T7-8. Axial MRI at the level of herniation demonstrating the spinal cord being pushed anteriorly herniating through the defect (C) as compared to normal spinal cord with cerebrospinal fluid space all around (B). 
later. A Duragen (Integra Life Sciences Corp., Plainsboro, NJ, USA) patch was used as graft to seal the defect which was reinforced with Tisseel fibrin glue. (Baxter Healthcare Corp., Deerfield, IL, USA) No fusion or instrumentation was performed. Patient experienced improvement in sensory symptoms right after the surgery and had improvement in her pain, motor strength $(4+/ 5$, MRC grade) and gait at three months follow-up with improvement in her Nurick myelopathy grade to 2. Postoperative MRI revealed reduction of the spinal cord with no evidence of herniation (Fig. 3).

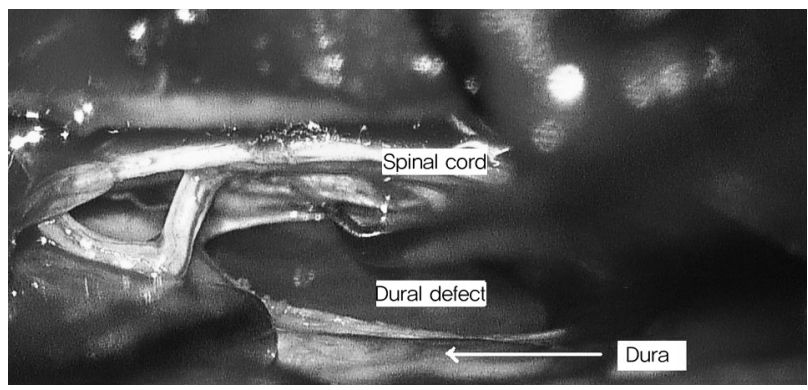

Fig. 2. Intraoperative photograph very clearly demonstrating the anterior dural defect with the reduced spinal cord.

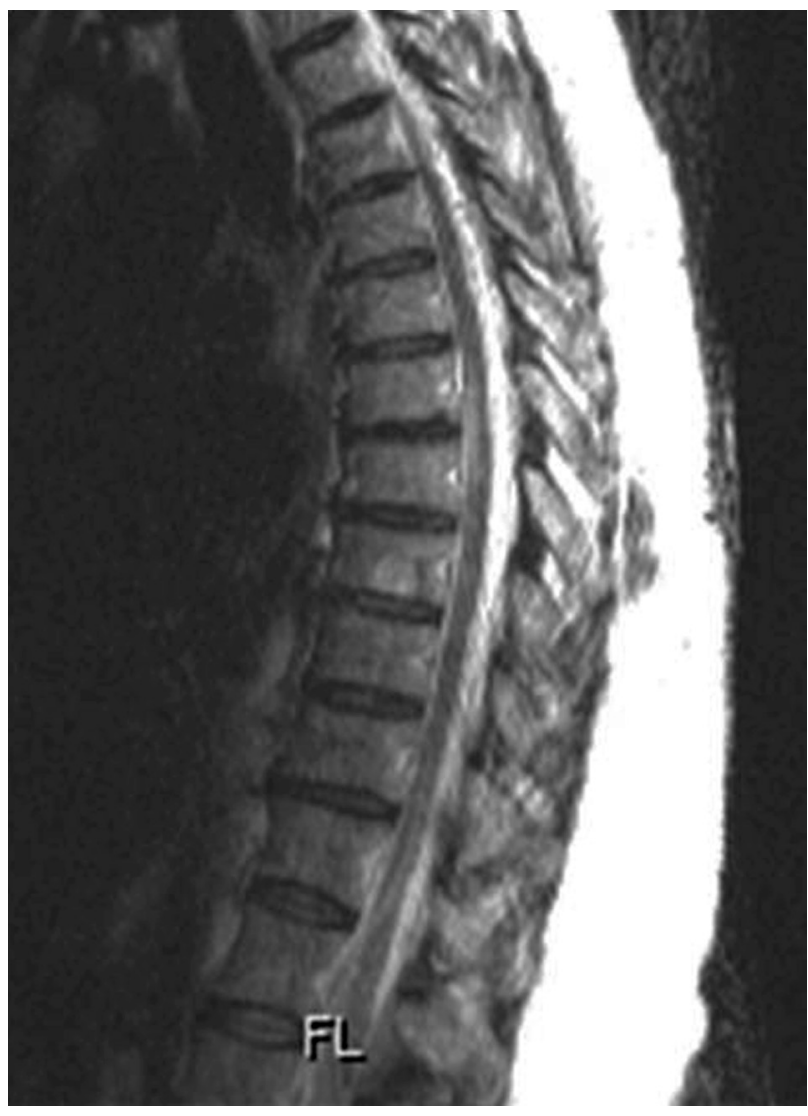

Fig. 3. Postoperative sagittal T2-weighted magnetic resonance imaging showing the reduced spinal cord with no evidence of herniation.

\section{Discussion}

Idiopathic ventral spinal cord herniation, a rare entity primarily affecting the thoracic cord was described first by Wortzman et al. in 1974 [5]. Improvement in imaging techniques has led to increased recognition of this entity; the fact that most of the cases are reported mainly in the last decade may indicate probability of missed diagnosis before the MRI era. Increasing awareness among the treating physicians is also a contributing factor towards increased cases being reported recently. The natural history of this condition is poorly understood as there are very few cases in the literature and most of them were treated surgically $[1,2]$. Massicotte et al. [3] reported four cases in their series that were managed expectantly in the absence of motor weakness and had no evidence of neurological deterioration at long term follow-up.

The exact etiology of this condition remains enigmatic with various postulated mechanisms. Congenital causes implicate the possibility of a congenital dural defect/duplication of the dura with defect in the inner layer or presence of dorsal arachnoid cysts which is not infrequently associated with this condition [1,2]. Similarly, acquired etiology points it towards damage to ventral dura mater secondary to inflammation, remote spinal trauma, or thoracic disc herniation $[1,2]$. Irrespective of the etiology, this condition occurs only in the thoracic cord; normal kyphosis of the thoracic cord and ventral position of the midthoracic cord being implicated for the same. The presence of dural defect causes a tethering of the spinal cord which is furthered aggravated by cerebrospinal fluid (CSF) pulsations and negative epidural pressures which leads to spinal cord damage from mechanical and vascular insult [1-9].

The tethering of the spinal cord at the level of the dural defect often results in progressive neurological symptoms which are often misdiagnosed. Brown-Sequard syndrome, spastic paraparesis, tethered cord syndrome, pure sensory or motor deficits are the various clinical presentations of spinal cord herniation [1-10]. Ventral thoracic spinal cored herniation's rarity often leads to delay in diagnosis which explains the long interval between onset of symptoms and surgical intervention (6 months to 36 years) reported in the literature [1].

The availability of MRI has facilitated the diagnosis of spinal cord herniation. A C-shaped kink typically seen on sagittal imaging is almost pathognomonic for this condition [3]. Spinal cord herniation has been classified as K, P 
and D type in a recent paper by Imagama et al. [7]. MRI feature can be missed by clinicians not treating intradural pathologies or misdiagnosed as an arachnoid cyst. There are numerous instances in the literature reporting re-exploration due to missed thoracic spinal cord herniation which was treated as posterior thoracic arachnoid cyst [2,9]. Dorsal arachnoid space is almost always enlarged in cases of spinal cord herniation and though, posterior arachnoid cyst may exist in isolation, neurosurgeons consider suspecting a ventral dural defect when operating such cases $[2,9]$. Computed tomography myelogram may be useful to evaluate the relationship of CSF to the spinal cord and it has been reported that in cases of idiopathic ventral herniation, there will be no filling defect dorsal to the spinal cord or retention of contrast agent ventral to the dura mater in contrast to arachnoid cysts which demonstrates an intradural filling defect posterior to the spinal cord [10]. Failure to recognize a spinal cord herniation may lead to biopsy of the normal but herniated cord with disastrous neurological consequences.

Surgery is the preferred treatment following diagnosis of thoracic cord herniation especially for patients with motor deficits or progressive neurological symptoms. Though described initially [5], ventral surgical approaches have been replaced by posterior approaches with dorsal laminectomy being the most common procedure to treat spinal cord herniation [1-4,7-10]. However, as the dural defect is located anteriorly, a surgical approach directed from the posterolateral aspect may provide more direct visualization of the dural defect with reduction of the spinal cord with less manipulation as compared to midline approach with laminectomy $[4,6]$. It also allows visualization both dorsal and ventral to the spinal cord and may allow treating/ruling out a concomitant arachnoid cyst. Paramedian unilateral transpedicular approach to treat spinal cord herniation was described by Gwinn and Henderson [6] with excellent clinical results and no complications. Similarly, Chaichana et al. [4] reported a bilateral transpedicular approach with unilateral removal of a pedicle and transverse process to provide a sufficiently wide bilateral exposure of the ventral dural defect [4]. We used a unilateral right paramedian transpedicular approach with excellent visualization of the dural defect without any spinal cord retraction or release of dentate ligaments. The selection of right versus left approach may be based on the location of the defect which may be eccentric to one of the sides. This approach may even facilitate use of tubular retractors for treating such cases which will further improve the overall surgical management of this increasingly recognized thoracic spinal cord pathology with further reduction in the overall morbidity. Use of intraoperative monitoring especially MEPs may be useful during the surgery $[2,4]$. SSEP monitoring is often unchanged because the ventral pathways are predominantly affected by the herniation. Enlargement of the ventral dural defect, primary closure of the defect with sutures, insertion of a ventral patch for duraplasty or use of graft with glue are the various options depending on the surgeons preference and expertise $[1,2]$. Though, suturing a graft seems appealing and is recommended by some surgeons, the superiority of one method over another is not demonstrated in any high quality study. Often, the defect is often very large, and it may not be technically feasible by all surgeons to suture a graft which may inadvertently lead to significant spinal cord manipulation with a potential to cause postoperative neurological deterioration. Groen et al. [2] reviewed cases of spinal cord herniation and concluded that widening of the dural defect is associated with higher chances of postoperative motor function improvement as compared to application of an anterior dura patch. BrownSéquard syndrome, severity of preoperative neurological deficits and release of the herniated spinal cord appeared to be strong independent factors, associated with favorable postoperative outcome highlighting the importance of early diagnosis and treatment [2,7]. We prefer laying a graft over the defect with reinforcement with glue as was performed in the case described. Surgery often results in good outcome in majority of cases; however, as patients with longer followup are available, cases of recurrence have started to surface which may refine treatment and lead to modification of the surgical strategy in the future [8]. The postoperative appearance after different surgical strategies and its role in follow-up is not well defined as only less than half of the cases reported in the literature described postoperative MRI features after reduction of spinal cord herniation [1,2].

To conclude, spinal cord herniation is unique to thoracic cord and is difficult to diagnose. As more and more clinicians are involved in treating patients with spine diseases, awareness of this entity is essential to avoid missing this potentially treatable condition. Considering the more frequent diagnosis with advanced imaging and increased recognition of this disease; newer surgical techniques, role of postoperative imaging, and long-term follow-up results will further facilitate management of this fascinating neurosurgical entity. 


\section{REFERENCES}

1. Shin JH, Krishnaney AA. Idiopathic ventral spinal cord herniation: a rare presentation of tethered cord. Neurosurg Focus 2010;29:E10.

2. Groen RJ, Middel B, Meilof JF, et al. Operative treatment of anterior thoracic spinal cord herniation: three new cases and an individual patient data metaanalysis of 126 case reports. Neurosurgery 2009;64(3 Suppl):145-59.

3. Massicotte EM, Montanera W, Ross Fleming JF, et al. Idiopathic spinal cord herniation: report of eight cases and review of the literature. Spine (Phila Pa 1976) 2002;27:E233-41.

4. Chaichana KL, Sciubba DM, Li KW, Gokaslan ZL. Surgical management of thoracic spinal cord herniation: technical consideration. J Spinal Disord Tech 2009;22:67-72.

5. Wortzman G, Tasker RR, Rewcastle NB, Richardson JC, Pearson FG. Spontaneous incarcerated herniation of the spinal cord into a vertebral body: a unique cause of paraplegia. Case report. J Neurosurg 1974;41:631-5.

6. Gwinn R, Henderson F. Transdural herniation of the thoracic spinal cord: untethering via a posterolateral transpedicular approach. Report of three cases. J Neurosurg Spine 2004;1:223-7.

7. Imagama S, Matsuyama Y, Sakai Y, et al. Image classification of idiopathic spinal cord herniation based on symptom severity and surgical outcome: a multicenter study. J Neurosurg Spine 2009;11:310-9.

8. Selviaridis P, Balogiannis I, Foroglou N, Hatzisotiriou A, Patsalas I. Spontaneous spinal cord herniation: recurrence after 10 years. Spine J 2009;9:e17-9.

9. Darbar A, Krishnamurthy S, Holsapple JW, Hodge CJ Jr. Ventral thoracic spinal cord herniation: frequently misdiagnosed entity. Spine (Phila Pa 1976) 2006;31:E600-5.

10. Uchino A, Kato A, Momozaki N, Yukitake M, Kudo S. Spinal cord herniation: report of two cases and review of the literature. Eur Radiol 1997;7:289-92. 